\title{
Histoarchitecture and Enzyme Profile Study in Interstitial Glands of Non Pregnant, Pregnant and Lactating Indian Leaf-Nosed Bat Hipposideros speoris (Schneider)
}

\author{
Dharna Bisen $^{1} *$ and Sharad Bisen ${ }^{2}$ \\ Department of Entomology, Department of Horticulture, College of Agriculture, \\ Balaghat, JNKVV, Jabalpur, India \\ *Corresponding author
}

\section{A B S T R A C T}

\section{Keywords}

Interstitial glands, Ovary, Pregnancy

\section{Article Info}

Accepted:

04 September 2019

Available Online:

10 October 2019
This study aimed to investigate the relation between interstitial glands, epithelial cords and pregnancy. The morphological and endocrine aspects of the ovarian interstitial glands of adult female bats were investigated to establish the probable function and the biological significance of this compartment in bats. Pregnant and non-pregnant adult female bats were used for the study. The females were classified according to their reproductive stages in inactive, active, pregnant, and post-lactating. The histological and histochemical features of the interstitial glands were studied. Interstitial glands showed variation in number and morphology during different stages of pregnancy. The cholesterol and its esters were present in non-pregnant females and were scarce in pregnant animals. Histochemical study of enzyme like glucose-6-

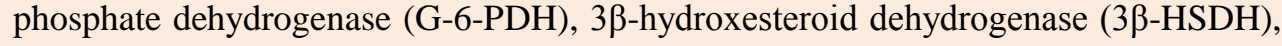
succinate dehydrogenase (SDH) and lipids showed variation at different stages of pregnancy. Our results suggest that the interstitial glands may be storage of precursor substances for the steroidogenesis. These precursors are probably used when the endocrine requirements are high, that is during the pregnancy. Thus this compartment may contribute to the normal gestation of bats. However, the relation between the interstitial cells epithelial cords and the pregnancy is complex, and further studies are needed to clearly establish it.

\section{Introduction}

In the order chiroptera, the patterns and strategies reproductive are diverse and consist of the most varied among mammals Peracchi et al., (2006). Four types of reproductive patterns are described for bats seasonal monoestry, seasonal polyestry, non-seasonal polyestry and bimodal seasonal polyestry (Fleming et al., 1972, Zortea 2003). The micro-chiropteran bat Hipposideros speoris was monoestrun bat and that feed on fruits and instect. Ovaries of placental mammals exhibit diversity of structure and function, although the pivotal role of producing ova and steroid hormones is common to all species 
chiropterans display variation of ovarian structure and functional adaptations that have few parallels. The interstitial glands of the ovary are not so well defined as those in the testis. Hipposideros speoris (order: chiroptera, suborder Microchiroptera and the family Rhinolophidae) is a seasonal breeder, monoesterous and monovular and show sinistral dominance. The mammalian ovary is a complex dynamic structure depicting conspicuous morphological, histochemical, biochemical and molecular changes involving follicles corpora lutea and interstitial gland cells during various reproductive states (Sastry and Pillai 2005, Dorlikar et al., 2013 and Godoy et al., 2014). Of the four major steroidogenically important component of the mammalian ovary viz-developing follicles, atretic follicles, corpora lutea and interstitial gland tissue (Igt), Igt are the most controversial and neglected component of the ovary and least studied. Three types of interstitial gland cells were observed in the ovaries of the bat Hipposideros speoris thecal, stromal and epithelial cords all of which exhibit cyclical variation histologically and histochemically in relation with reproductive cycle (Parrott and Skinner 2000, Sastry et al., 2005, 2008, 2010; Singh et al., 2005, Gill et al., 2007 and Trivedi and Lall 2007). We choose to study histochemical localization of SDH, G-6-PDH, 3 $\beta-\mathrm{HSDH}$ and lipids to examine the site and changing pattern of its activity in ovaries of Hipposideros speoris during non-pregnant and pregnant states. The purpose of the present study provides a brief account on interstitial gland cells and its role in steroidogenesis during different stages of reproduction.

\section{Materials and Methods}

Collection of animals and histology :- All experiments were conducted in accordance with the principles and procedures approved by the Departmental research committee,
RTM University Nagpur, Maharastra, India. More than 20 specimens of Hipposideros speoris were collected once in a calendar month with the help of mist net from natural population inhabiting abandoned mines in Khapa (2092'N, 78 95'E) Nagpur, Maharashtra throughout the reproductive cycle. These species are found to inhabit in cold and humid places preferably dark. All the bats from the same colony do not show same pattern of reproductive behavior because there exists an asynchrony in reproductive cycle among different females of the same colony. For histological studies the ovaries were fixed quickly in Bouin's fixative, dehydrated in ethanol and embedded in paraffin wax. The sections were cut at $5 \mu \mathrm{m}$, stained with haematoxylin and eosin.

\section{Histochemical detection of lipids}

Ovaries fixed in formol-calcium were cut on freezing microtome at $-20^{\circ} \mathrm{C}$ and were stained by Chiffle and Putt method (Lillie and Fullmer, 1976). These cryocut sections $(10 \mu \mathrm{m})$ thick were washed briefly in water and stained with Sudan black B for general lipids. Lipids appeared black or bluish black.

\section{Histochemical detection of enzymes}

For histochemical localization of

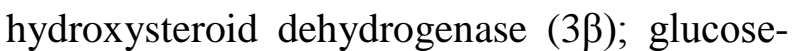
6-phophatase dehydrogenase (G-6-PDH) and succinic dehydrogenase $(\mathrm{SDH})$ sucrose fixed tissues were cut on cryostat $\left(-20^{\circ} \mathrm{C}\right)$ at $10 \mu \mathrm{m}$ thicknesses. The incubation medium of $3 \beta$ HSD and G-6-PDH consisted of nitroblue tetrazolium (nitro BT), Nicotinamide adenine dinucleotide $\left(\mathrm{NAD}^{+}\right)$and pregnenolone dissolved in 2-2 dimethylformamide for $3 \beta$ HSD. For G-6-PD the substrate was glucose6-phosphate dissolved in 2-2 dimethylformamide. The sections were incubated in substrate media (di-sodium succinate) for SDH. Enzyme product was 
visualized by conversion of nitro BT (nitroblue tetrazolium) to tetrazolium granules. Appropriate controls were run in substrate deficient media. The reaction product colour intensity was visually scored as $+++=$ strong, $++=$ moderate, $+=$ low, $-=$ negligible, $--=$ no reaction $($ Table- 1 ).

\section{Results and Discussion}

The appearance of thecal interstitial gland tissue observed in this study during pregnant and non-pregnant phases as described in other bats, Rhinopoma microphyllum kinneari (Trivedi and Lall, 2004; 2007); however, without specifying the reproductive status of the ovary, origin, development and functional status notable to clarify the role of these glands (Singh et al., 2005). The thecal type interstitial cells originate due to hypertrophy or transformation of theca interna of atretic vesicular and multilaminar follicles in Hipposideros speoris (Fig. 4 and 10). The thecal Igc are of very transient nature in Hipposideros speoris (Fig. 1, 7 and 13) as in few other cases such as women, rhesus monkey, cow and buffalo as they quickly revert to the embryonic stromal. Besides reverting back to the original stromal tissue, some interstitial tissue in Hipposideros speoris were also observed to undergo degeneration.

The presence of varying amounts of diffuse lipids and lipid droplets in the interstitial tissue signifies its importance as steroidogenic tissue, as lipid serves as the potential precursor material for steroid biosynthesis (Fig. 16, 19 and 22).

The thecal interstitial glands of Hipposideros speoris exhibits a positive reactivity for steroidogenic enzymes 3 $\beta$-HSDH (Fig. 16, 19 and 22), SDH (Fig. 34, 37 and 40) and G-6PDH (Fig. 43, 46 and 49) during inactive and active. Such enzyme activities, indicative of steroid synthesis have been demonstrated in the interstitial gland cells of thecal origin of some bats (Singh and Krishna, 1994; Singh et al., 2005 Trivedi and Lall, 2004, 2007). The foregoing observations conclude that the process of atresia in the antral and secondary follicles (Fig. 1) in Hipposideros speoris appears to be related to the formation of thecal type interstitial gland cells, which maintains ovarian integrity via bio-synthesis, growth rate of follicle, selection of dominant follicle which happened to be one in Hipposideros speoris, as the bat is monovular and monoeastrus (Novaldo et al., 2018, Young and McNeilly 2018).

Both morphological and histochemical studies reveal that stromal Igc in $H$. speoris are better equipped for steroidogenesis (Fig. 2, 5 and 11). The histological changes seem to be associated with the histochemical activities of the stromal interstitial cells (Parrott and Skinner 2000). The intensity of the enzymes 3ß-HSD (Fig. 32, 35, 38, 41), G-6-PD (Fig. 55, 56 and 57), SDH (Fig. 44, 47, 50 and 52) and lipid (Fig. 20, 23, 26 and 29) accumulation in stromal interstitial cells in the ovary of Hipposideros speoris showed variations during the active, pregnant and lactational phases. Increased lipid accumulation during inactive phase could be due to the sharp decline in androgen production.

All these observations also strongly support the suggestion that the lipid droplets in the Igc are the stores of potential precursor materials which are converted into steroid hormones when the proper gonadotrophic stimulation becomes available. So far, the ovarian stroma (or Igc) has been believed to form mainly androgens (Guraya, 2000) estrogens (Singh and Krishna, 1994; Singh et al., 2005) and progestins (Trivedi and Lall, 2007 and Godoy et al., 2014). However, in the present work the observations on the annual reproductive cycle of the bat, $H$. speoris emphasizes the synthesis of androgens (Fig. 1-50). 
Table: 1 The cellular localization and relative activity of different histochemical stains in the interstitial gland (thecal,stromal and epithelial cords) are shown in table-1 correlative to its development only during different phases of reproductive cycle in the ovary.

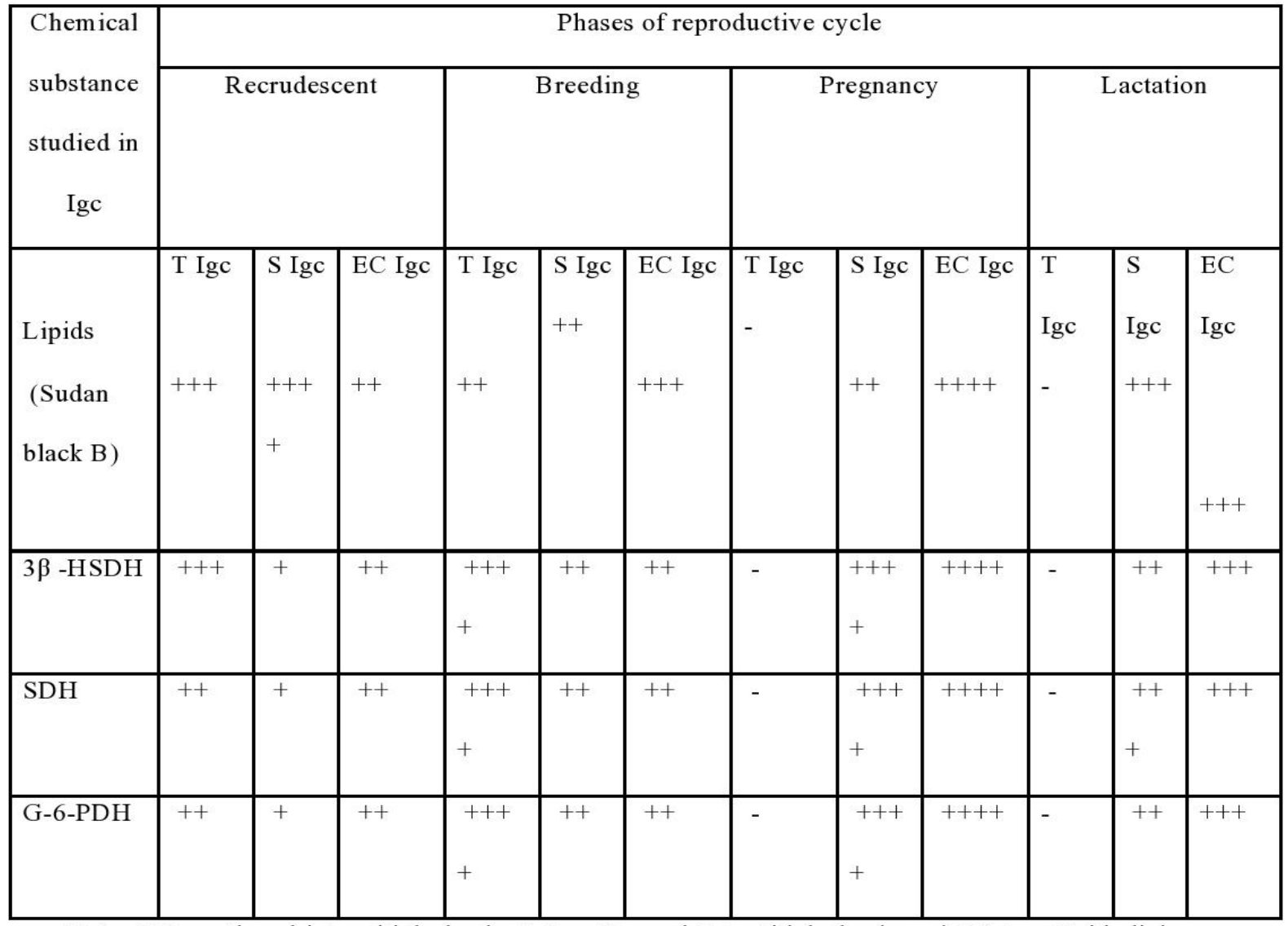

Note: T Igc : thecal interstitial glands, S Igc: Stromal Interstitial glands and EC Igc: Epithelial cords 

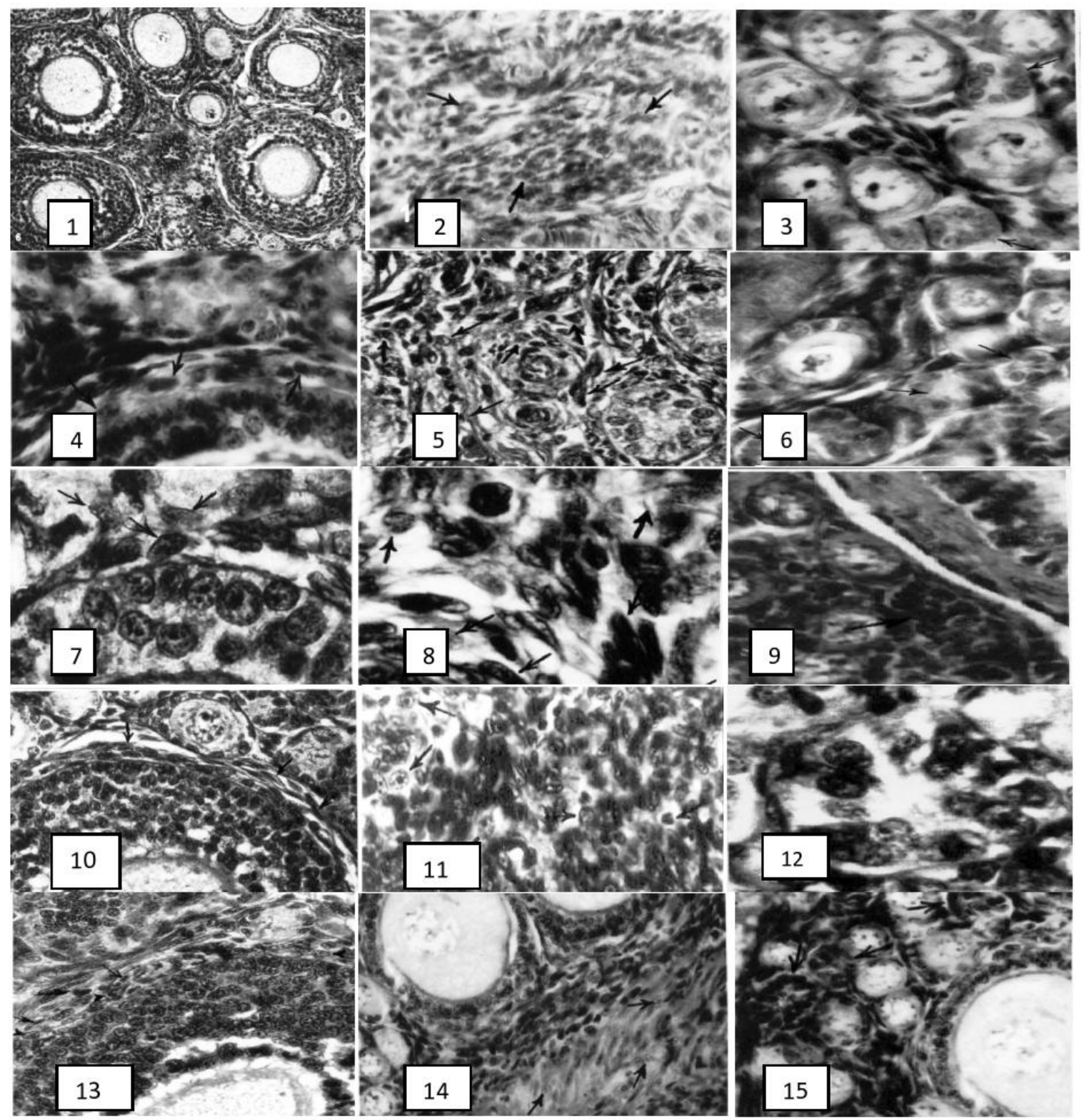

Plate 1: Histological photos showing interstitial glands of reproductive cycle : (Fig. 1-15) Fig.1.Initiation of thecal gland formation inactive ovary. X250. Fig.2. Spindle-shaped stromal cells compactly arranged in the medulla of inactive ovary.X 400 . Fig.3 . Reduced but well-defined cords at early active phase X400. Fig.4. Thecal interstitial gland is markedly distinguishable (arrow) from late active phase X 450. Fig.5. The medullary region showing 2-3 small spherical Igc with indistinct nuclei (arrow) from late inactive ovary X 400. Fig.6. Epithelial cords (arrow) distributed individually between follicles at late-active X400. Fig.7. Further increase in the thickness of thecal interstitial band with an increase in the number of cells during active X1000. Fig.8. Late active ovary showing well developed and distinct stromal Igc in the cortical region (arrow) X1000. Fig.9. Part of the ovarian cortex from early pregnancy to show few epithelial cords X400. Fig.10. Part of ovary from (late-active phase) differentiation and development of thecal interstitial gland is of significance (arrow) X450. Fig.11. Note section of ovary displaying the rounded-shaped similarly hypertrophied stromal Igc during mid-pregnancy (arrow) X400. Fig.12. Increase in the number of cords, with simultaneous increase in cell-number (15-16) in each cord during pregnancy. X1000. Fig.13. Thecal interstitial gland cells showing regression (arrow). Fig.14. Lactational ovary showing declined number of stromal Igc (arrow) X400. Fig. 15.Recycle of epithelial cords occur with the approach of lactation exhibiting some degenerative EC (arrow)X400. 

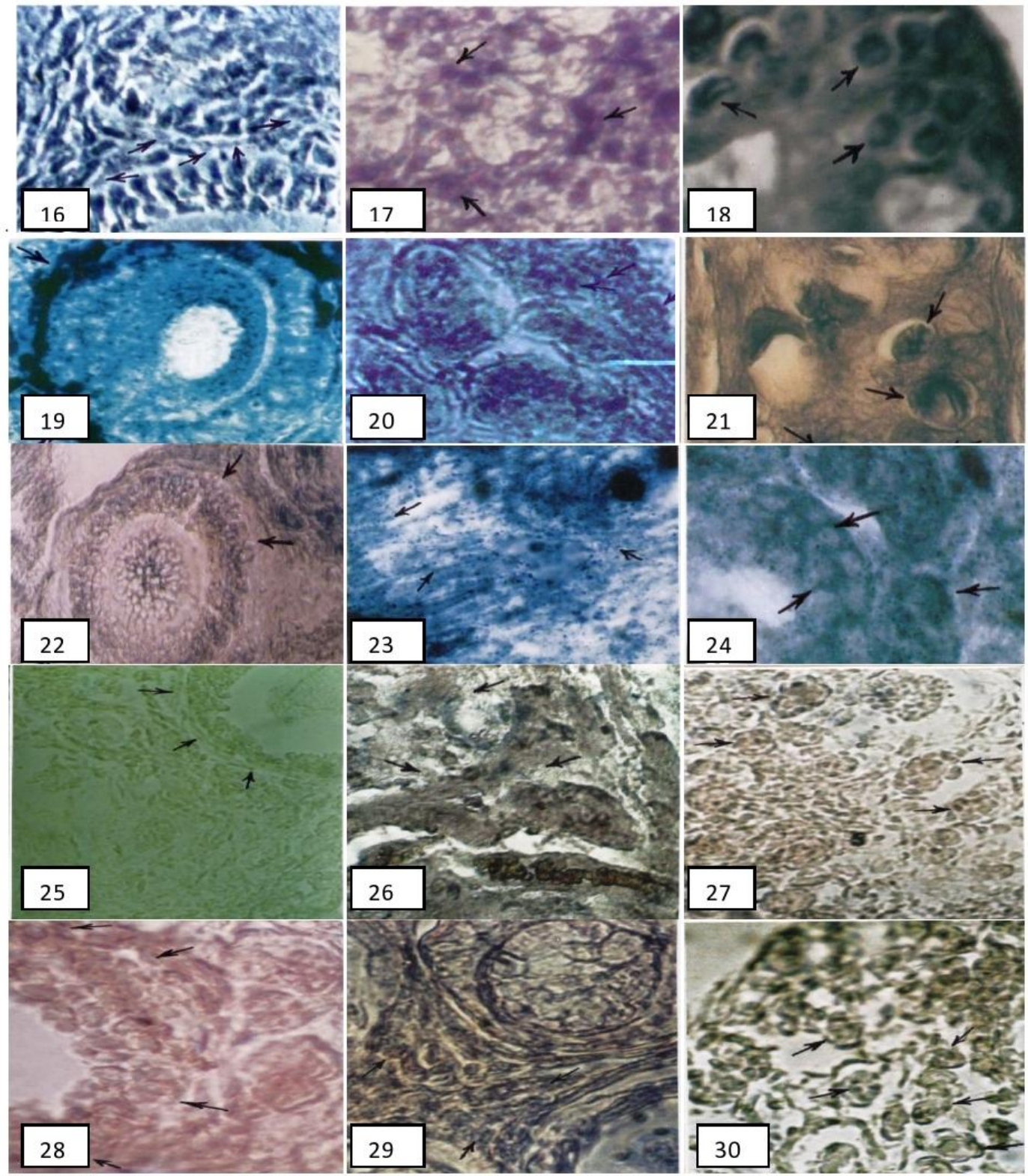

Plate 2: Histochemical photographs showing staining profile in interstitial glands of reproductive cycle:(Fig.16-30): Fig.16. Arrow marks the developing theca having negligible deposition of lipid (early (arrow) inactive phase) X1000. Fig.17.The heavy deposits or droplets of variable but of relatively larger size in the stromal Igc during inactive phase X400. Fig. 18.Small-sized inactive epithelial cords from inactive ovary demonstrate for lipids (arrow) X400. Fig.19.Comparabl noticeable moderate lipid activity (arrow) during acive phase in the theca X400. Fig.20. Note lipid droplets during late inactive period which appear strongly positive (arrow) X400. Fig.21.Few cords (arrow) evident in the early active ovary showing lesser amount of lipid droplets.X100. Fig.22.Apparently no reactivity noticed during (late- active) as thecal cells undergo degeneration (arrow) X400. Fig.23.Note lipid activity increase during active.X400. Fig.24.Active phase ovary showing some epithelial cords with lipid accumulation of moderate intensity (arrow) X 100. Fig.25. Thecal gland (arrow), from early active phase exhibiting faint $3 \beta$ HSDH reaction X400. Fig. 26. During advance pregnancy the stromal Igc for sudanophilic reactivity appears negligible (arrow) X400. Fig.27. Epithelial cords from early pregnancy coinciding the implantation of blastocyst demonstrating low intensity lipid droplets along the cell outlines (arrow) X 450.Fig.28. Formazan granular deposition in thecal cells as approaches towards pregnancy. X1000. Fig.29.Lactational ovary stromal Igc are less in number but intensity is slightly decreased (arrow) X400. Fig. 30. Few small epithelial cords from lactating ovary showing reduced lipid droplets along the cellular borders (arrow) X 450 . 



Plate 3: Fig.31.Note during early (late inactive period) thecal gland are undergoing degeneration hence demonstrate low profile

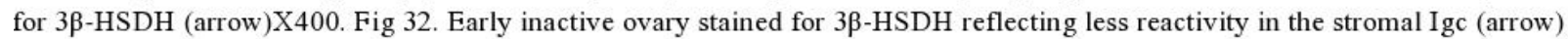

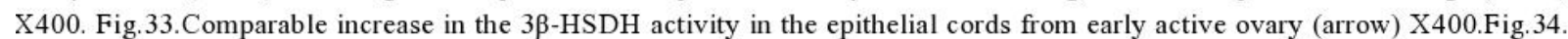
Slight in SDH activity is detectable in theca during active stage (arrow)X400.Fig.35.Note few granule deposition in stromal Igc during late-inactive (arrow)X400. Fig.36. Reduction in the size of epithelial cords correspond to active period, however, an enhancement of staining profile occurs (arrow)X400. Fig.37.Note thecal interstitial gland exhibiting higher intensity for SDH during November corresponding to active phase (arrow) X400.Fig.38.W th the advancement of pregnancy the intensity of $3 \beta$ -

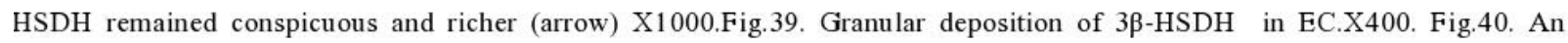
ovarian section from late active show low SDH activity in theca layer (arrow)X400.Fig.41.Decrease in enzyme activity as approaches towards lactation.X400.Fig.42 Note an abrupt shoot up in the 33-HSDH reactivity in highly developed epithelial cords (arrow) during mid-pregnancvX250. Fig.43. An antral follicle from earlv Sedtember show developing theca with negligible activity for G-6-PD(arrow)X450.Fig.44.Peripheral region of the late-active depicting positive SDH reactivity in stromal cells(arrow)X400Fig.45.3B-HSDH reactivity enhances in the lactational ovary with moderate to high activity(arrow) X 100 . 


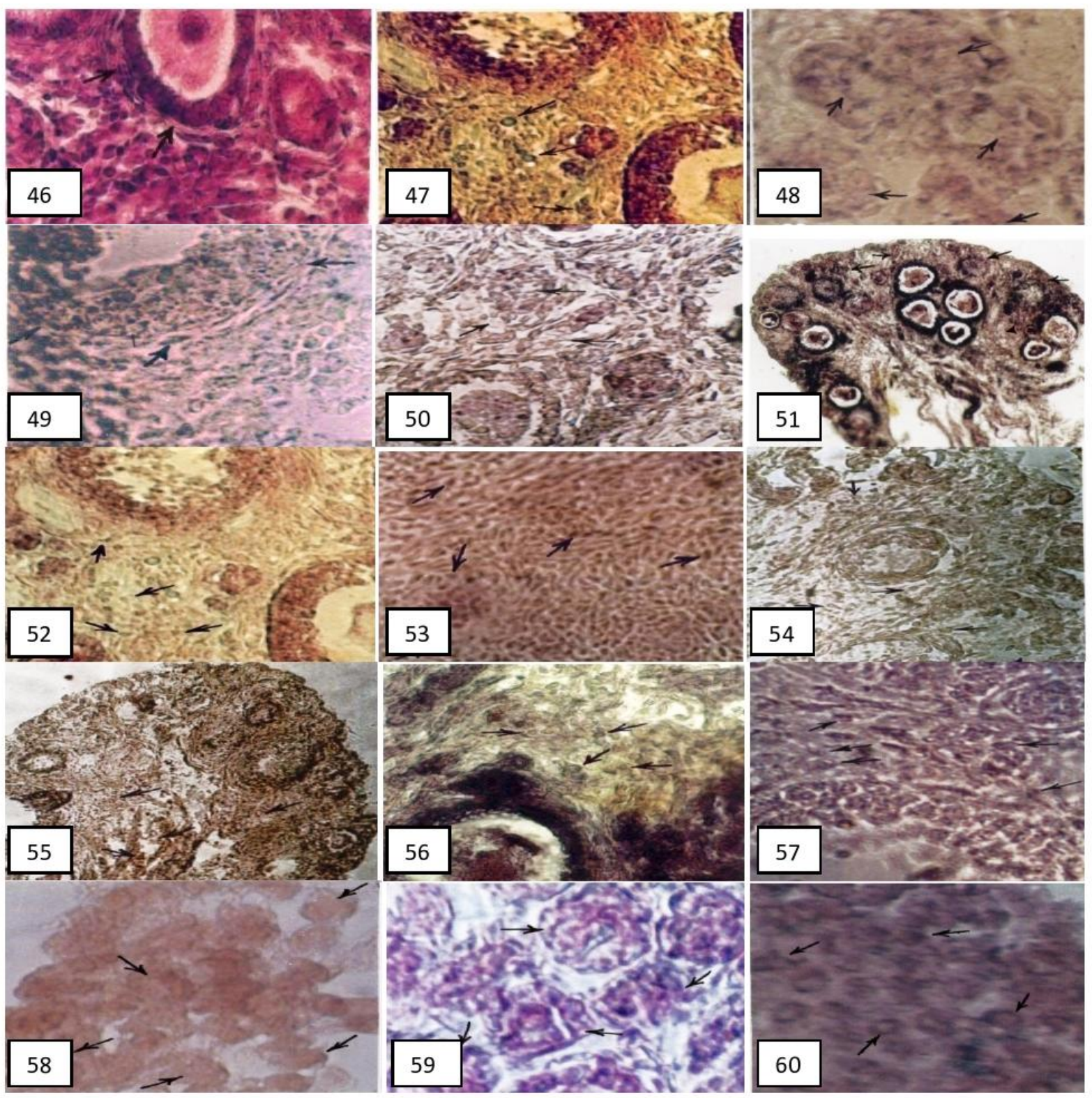

Fig.46.Arrow marks the thecal interstitial gland stained for SDH during active phase.X400. Fig. 47. Inhance in SDH activity as approaches towards active phase.X400. Fig.48 Irregularly distributed small sized cords in early active ovary having some granular deposition for succinate dehydrogenase enzyme (arrow) X400. Fig.49.Regressed thecal Igc (arrow) demonstrating depletion in G-6-PDH reactivity from early active period. X450. Fig.50. The reaction is concentrated particularly in stromal cells for succinic dehydrogenase in the advance stage pregnant ovary X400. Fig.51. Note greater concentration of SDH granules of succinate dehydrogenase from pregnancy in cords (arrow) X 400. Fig.52. Stromal Igc from lactation exhibiting low to moderate SDH granules (arrow) X400. Fig. 53. Ovary from lactational displaying granular deposition in cords static to previous picture (arrow) X400. Fig 54..Peripheral region of the inactive ovary displaying moderately stained (G-6-PDH) interstitial epithelial cords (arrow) X100. Fig. 55. Ovary at active phase stained for G-6-PDH. Note low to moderate stained stromal Igc in the medullary region (arrow) X100.Fig. 56. G-6-PDH activity in the stromal Igc is at spurt during advanced pregnancy (arrow) X1000. Fig. 57. High power view from lactational ovary displaying depletion of the formazan granules in stromal Igc (arrow) 
The present study also emphasizes that cyclic changes in the interstitial epithelial cords (EC) are related to the reproductive cycle of Hipposiders speoris. No one can noticed this type of interstitial gland cells earlier in bats (Gill 2007 and Trivedi and lall 2007). We observed these Igc cells first in Hipposiders speoris.

During mid-pregnancy, they were observed to be highly hypertrophied, closely clustered in the cortical portion of the ovary (Fig. 9), each with 20-30 hypertrophied cells in each cord. Early inactive phase the chords are small and inactive and there was a sudden burst in $\mathrm{SDH}$, $3 \beta$-HSD and G-6-PDH staining profile.

Through the activity of SDH is used as a criterion of luteal function, its activity is correlated with presumed sites of hormone production and places of cellular proliferation (Sastry and Tembhare 2008, 2009, Sastry et al., 2010) However G-6-PDH activity was at more elevated levels during advanced pregnancy as evident from the occurrence of density populated, highly hypertrophied zones in the cortex (Gill et al., 2007, Dorlikar et al., 2013, Zahra and Farangis 2015, Nivaldo et al., 2018, Sastry and Pillai 2005, 2008).

Thus we suggest that this compartment may be a storage precursors for the steroidogenesis and then, the precursors are principally used during pregnancy by other ovarian compartments (e.g.corpus luteum) when the endocrine requirements are higher.

The observed histological and histochemical feature of Igc suggest steroidogenic activity, thus these cells may contribute to the total endocrine production synthesized by the ovary. Then the Igc of Hipposiders speoris may play a role as a source of steroid precursors during pregnancy and probable further in its maintenance. However, further studies are necessary to conform their roles.

\section{References}

Dorlikar, A.V., Dhamani, A.A., Charde, P.N., Mohite, A.S. (2013). Morphomet ric and histoarchitectural changes phases of reproductive cycle. International Journal of Molecular Zoology, 3: 3241.

Fleming, T.H., Hooper, E.T., Wilson, D.E., (1972). Three central American bat communites: structure, reproductive cycles and movement patterns. Journal of Ecology 53:555-569.

Ghobadian, Z. and Ghassemi F. (2015) The ovary of fruit bat Rossetus aegyptiacus (Megachiroptera: pterpodidae) in southern Iran. Journal of Zoology 4 (1):57-61.

Gill, E., Forneris, M., Dominguez, S., Penissi, A., Fogal, T., Piezzi, R.S., Scardapane L. (2007). Morphological and Endocrine study of the Ovarian Interstitial Tissue of Viscacha (Lagostomus maximus maximus). Journal of Anatomical Record, 290:788-794.

Godoy, M.S.M., Carvalho, W.D., Esberard, C.E.L. (2014). Reproductive biology of the bat Sturnira lilium (Chiroptera, Phyllostomidae) in the Atlantic forest of Rio de Janerio, southeastern Brazil. Journal Brazil Biology, 74(4):913-922.

Guraya, S.S. (2000). Comparative Cellular and Molecular Biology of Ovary in Mammals: Fundamental and Applied Aspects, Oxford and IBH Publishing Co., Pvt. Ltd., India, pp. 195-236.

John, J., Rasweuker, V., Nilima, K., Badwaik, R., Kiranmayi, V., Mechineni, S. (2011). Ovulation, Fertilization, and Early Embryonic Development in the Menstruating Fruit Bat, Carollia perspicillata. Journal of Anatomical Record, 294:506-519

Lillie, R.D. and Fullmer, H.M. (1976). Histopathologic Technic and Practical 
Histochemistry. $4^{\text {th }}$ Edition McGrawHill Book Company. New York.

Novaldo, B., Maria, J.G., Fabricya, R.S., Erivaldo, A.A., Francisco, C.A., Ahearo, A.C., Jose, E., Katharine, R.P. (2018). Analysis of the reproductive stage and ovaries histomorphometry of Dermanura cinerea (Chiroptera: phyllostomidae) in an Atlantic forest fragment of Pernambaco, northeastern. Journal of Pesquisa Veterinária Brasileira 38(1): 167-174.

Parrott, J.A. and Skinner, M.K. (2000). Kit ligand on ovarian stromal cells: effects on theca cell recruitment and steroid production. Journal of Molecular Reproduction and Development. 55: 55-64.

Peracchi, A.L., Lima, I.P., Reis, N.R., Nogueira, M.R., Ortencio, F.H. (2006). Ordem chiroptera, In: Reis NR, Peracchi AL, Pedro WA and Lima IP. (Eds), Maniferos do Brasil. Edifurb, Londrina. P. 153-230.

Pillai, S.B. and Sastry M.S. (2012). A mini review of female reproductive asymmetry in microchiropteran bats with a special approach to the leafnosed bat, Hipposideros speoris

Journal International Current Science, 4: 96-111.

Sastry, M.S. and Pillai, S.B. (2005). Cyclical changes in the epithelial cords of ovary of a microchiropteran bat, Hipposideros speoris (Schneider). Journal of Endocrinol and Reproduction, 9: 37-42.

Sastry, M.S. and Tembhare, D. (2008). Some histochemical observations on the ovarian stromal interstitial cells during anoestrous and oestrous in Indian leafnosed bat Hipposideros speoris (Schneider) Journal The Bioscan,3(2):139-146.

Sastry, M.S. and Pillai, S.B. (2008). Variations in the epithelial cords of ovaries of microchiropteran bat, Hipposideros speoris (Schneider) during reproductive cycle: an enzymatic approach, Journal of Theoretical and Experiment, 4: 95101.

Sastry, M.S. and Tembhare, D. (2009). Histochemical study of ovarian dehydrogenases SDH and Glucose-6phosphate during various phases of reproductive cycle in Indian leaf-nosed bat Hipposideros speoris (Schneider). Journal The Bioscan, 4(1):45-51.

Sastry, M.S., Pillai, S.B. and Tembhare, D. (2010). Histochemical analysis of the thecal interstitial gland in the ovaries of the microchiropteran bat, Hipposideros (Schneider) during the reproductive cycle. Journal of Endocrinol Reproduction, 14 (2): 6572.

Singh, U.P. and Krishna, A. (1994). Seasonal changes in the cytochemical and ultrastructural features of the ovarian interstitial tissue in a vespertilionid bat, Scotophilus heathi. Journal of Reproduction Biology and Comparative and Endocrinology, 6(1): 33-46.

Singh, U.P., Krishna, A., Smith, T.D., Bhatnagar, K.P. (2005). Histochemicallocalization of enzymes and lipids in the ovary of a vespertilionid bat Scotophilus heathi during the reproductive cycle. Journal of Brazilian Zoology. 65: 179-186.

Trivedi, S. and Lall, S.B. (2004). Ovarian dehydrogenases of the non-pregnant, pregnant and lactating Rhinopoma microphyllum kinneari (Chiroptera: Rhinopomatidae). Vespertilio. Journal of Animal Biology, 8: 105-112.

Trivedi, S. and Lall, S.B. (2007). Histoarchitecture and $1-3 b$ hydroxysteroid dehydrogenase profile in ovaries of the non-pregnant, 
pregnant and lactating insectivorous rat-tailed bat, Rhinopoma microphyllum kinneari. Journal of Animal Biology. 57: 97-114.

Young, J.M. and McNeilly, A.S. (2018) Theca: the forgotten cell of the ovarian follicle. Journal of Society for
Reproduction and Fertility (paper):1741-7899.

Zortea, M. (2003). Reproductive patterns and feeding habits of three nectarivorous bats (Phyllostomidae: Glossophaginae) from the Brazilian Cerrado. Journal of Brazilian Biology. 63(1):159-168.

\section{How to cite this article:}

Dharna Bisen and Sharad Bisen. 2019. Histoarchitecture and Enzyme Profile Study in Interstitial Glands of Non Pregnant, Pregnant and Lactating Indian Leaf-Nosed Bat Hipposideros speoris (Schneider). Int.J.Curr.Microbiol.App.Sci. 8(10): 324-334.

doi: https://doi.org/10.20546/ijcmas.2019.810.033 